\title{
Application of matched asymptotic expansion techniques to the analysis of geothermal heat exchangers
}

\author{
Miguel Hermanns
}

\author{
Santiago I báñez
}

\begin{abstract}
Most theoretical models for the thermal response of geothermal heat exchangers assume the mean azimuthal borehole wall temperature to be uniform along the boreholes. This simplifying assumption, closely related to the g-functions introduced by Eskilson in 1987, has dominated the research field for the past 30 years, allowing the analysis of large geothermal heat exchangers in reasonable amounts of time. The assumption, however, is not physically correct, which binders the attainable accuracy. By using matched asymptotic expansion techniques, analytical models for the thermal response of geothermal heat exchangers are derived, which do not require the aforementioned simplification. The resulting expressions, applicable to geothermal heat exchangers with irregularly placed heterogeneous boreholes, show accuracy and flexibility levels comparable to SBM, but with a computational cost in line with the use of g-functions.
\end{abstract}

\section{NTRODUCTI ON}

Geothermal heat exchangers have an increasingly fundamental role in the design and construction of sustainable buildings. An HVAC system based on geothermal energy can only be considered truly renewable, however, if the geothermal heat exchanger and the heat injection/extraction strategy are properly designed. Otherwise, thermal exhaustion of the ground occurs, and the geothermal heat pump's efficiency decreases over time.

To ensure the long-term efficiency of geothermal HVAC systems, its thermal behavior after 25, 50, or even 100 years of operation must be known, reason why their theoretical and numerical modeling is extremely relevant to the industry. Extensive literature exists on this topic, being the contributions by the research group at Lund University the most influential ones (Bennet et al. 1987; Claesson and Hellström 2011; Eskilson 1986, Eskilson 1987; Eskilson and Claesson 1988; Hellström 1991). They proposed two models. The Superposition Borehole Model (SBM) solved numerically the governing equation in the ground and the one-dimensional energy conservation equations for the transfer of heat along the pipes. In order to connect both, a network of thermal resistances was used to describe the quasi-steady twodimensional thermal response of the grout and of the ground close to the borehole (Eskilson 1986). The extension to borehole fields was done by superposing the exerted temperature perturbation of neighboring boreholes. This approach rendered the model very accurate but also very slow, especially for large borehole fields. The second one, the g-function model, assumed a uniform mean azimuthal borehole wall temperature along the boreholes (Eskilson 1987). This simplification allowed the uncoupling of the heat conduction problem in the ground from the heat transfer problem inside the boreholes. For the former problem, a large database of precomputed borehole field configurations, the so called g-functions, was obtained using a tailored version of SBM, while the latter problem was solved analytically thanks to the introduced simplifying assumption. The result was a less accurate and less flexible model than SBM, but an extremely fast one. 
A lot of effort has been made to develop efficient but accurate methods to calculate the $g$-functions (Li and Lai 2015). In recent years, g-functions have been obtained using a commercial finite-element simulation tool (Monzó et al. 2016), but this approach is very time consuming. On the other hand, a family of models assimilates the borehole to a line of heat sources of uniform intensity (Eskilson 1987). The infinite line source (ILS) (Ingersoll et al. 1954), the infinite cylindrical-surface source (ICS) (Ingersoll et al. 1954) and the finite line source (FLS) (Zeng et al. 2002; Lamarche and Beauchamp 2007) models have been used respectively to model the borehole for increasing values of the characteristic injection time. All these models assume that the heat injection rate per unit borehole length $q$ is uniform along the borehole, contrary to the original g-function definition proposed by Eskilson that assumes a uniform mean azimuthal borehole wall temperature $T_{b}$ along the borehole. As the resulting value of $T_{b}$ is not uniform along the borehole, its mean value is used instead to define the g-functions (Zeng et al. 2002; Lamarche and Beauchamp 2007; Claesson and Javed 2011; Cimmino and Bernier 2014; Cimmino et al. 2013) and its calculation can be reduced to a single integral (Lamarche and Beauchamp 2007; Claesson and Javed 2011) that is discretized using the midpoint rule with one (Cimmino et al. 2013) or more segments (Cimmino and Bernier 2014; Lazzarotto and Björk 2016) or the trapezoidal rule with multiple segments along the boreholes (Lamarche 2017). Furthermore, a primitive function for the integral can be obtained for the steady-state and time-harmonic cases (Hermanns and Ibáñez 2018) leading to simple analytical expressions. This alternative $g$-function definition results in very efficient methods. However, discrepancies up to $30 \%$ have been reported between Eskilson's original definition with uniform $T_{b}$ and the alternative definition with uniform $q$ (Cimmino and Bernier 2014; Malayappan and Spitler 2013) that grow as the number of thermally interacting boreholes increases (Cimmino and Bernier 2014; Cimmino et al. 2013).

Those two simplifications that assume uniform values along the borehole are inherently limited in accuracy as shown in Malayappan and Spitler (2013), Hermanns and Ibáñez (2017), and Ibáñez and Hermanns (2018) among others. Besides these accuracy problems, the mathematical problem that the original Eskilson's definition of the g-function gives rise to, becomes ill-posed under certain circumstances (Hermanns and Ibáñez 2018). Therefore, a well-posed theoretical model that can retain axial variations of the mean azimuthal borehole wall temperature and the heat injection rate per unit borehole length is needed.

Using matched asymptotic expansion techniques (Lagerstrom 1988), the leading author and his research group have derived analytical expressions for the thermal response of geothermal heat exchangers without requiring the aforementioned simplifications. The resulting model is comparable to SBM in terms of flexibility and accuracy, but with a computational cost in line with the g-function model. Despite being also an analytical method, the proposed method clearly differs from the g-function model in that the thermal responses of the pipes and the liquid inside it, the grout, and the ground, all are tackled at once without assuming neither $T_{b}$ nor $q$ to be uniform along the borehole, that is, the developed solutions directly give the thermal response of the geothermal heat exchanger.

The present conference paper is a summary of the work done so far by the leading author and his research group. First, a description of the characteristic times that appear in the problem is given. The resulting inner and outer regions and the asymptotic matching process that weaves them are then described. An overview of the process carried out to obtain an asymptotic solution is given afterwards. The extension of the proposed model from one to multiple boreholes is discussed here for the first time. The proposed model is compared against existing models for a test case of a timeharmonic sub-annual excitation applied to a borehole field comprised by two coaxial probes of different lengths. These preliminary results state clearly the differences between the g-function model and the asymptotic model developed by the authors. Finally, conclusions and indications on the expected achievements to accomplish in the coming years are given.

\section{SLENDER GEOTHERMAL BOREHOLES}

A vertical geothermal borehole consists of a set of pipes placed in a vertical drilling, filled with grout, and through which a liquid flows and exchanges heat with the surrounding ground. Typical boreholes present depths $H$ of the order of hundreds of meters and radii $r_{b}$ of the order of tens of centimeters, leading to aspect ratios $\Lambda \sim H / r_{b}$ of the order of thousands. This extreme slenderness is responsible for the appearance of two distinct regions, an inner one located at 
radial distances to the borehole of order $r_{b}$, and an outer one located further away. To obtain the thermal response of the borehole, the solutions to these two regions need to be combined, something that is accomplished through asymptotic matching at an intermediate scale (Lagerstrom 1988).

Closely related to the previous two length scales are the characteristic transversal diffusion time, $t_{b} \sim r_{b}^{2} / \alpha$, and the characteristic longitudinal diffusion time, $t_{H} \sim H^{2} / \alpha$, where $\alpha$ is the thermal diffusivity of the ground. Considering the typical values attained by the different parameters, these two characteristic times are respectively of the order of hours and centuries (Hermanns and Pérez 2014). Two additional characteristic times can be identified in the problem. The first one is the residence time of the liquid in the pipes, $t_{r} \sim V / H$, with $V$ being the mean flow velocity of the liquid. Due to the requirement of turbulent flow regime to enhance the heat exchange between the liquid and the pipe wall, this characteristic time is of the order of tens of minutes, so that $t_{r} \ll t_{b} \ll t_{H}$ (Hermanns and Pérez 2014). The second one is the characteristic heat injection time $t_{q}$. Since the heat injection/extraction from the ground depends on the cooling/heating needs of the building, this characteristic time varies from minutes to months. Also, characteristic values of years or even decades are common, as certain heat injections/extractions are sustained over time.

The thermal problems to solve in the inner and outer regions depend on the relationship between $t_{q}$ and the other three characteristic times. The following discussion will focus on the realistic case in which $t_{r} \ll t_{b} \ll t_{q} \ll t_{H}$. For the sake of simplicity, the presentation will be limited to simple U-pipe or coaxial pipe configurations with two pipes connected at the bottom, one with the flow downwards (pipe 1) and one with the flow upwards (pipe 2). A straightforward extension to other borehole configurations, like double U-pipes and energy piles, is possible if certain thermal symmetries are preserved (Hermanns and Ibáñez 2017; Ibáñez and Hermanns 2018).

\section{Inner region}

The inner region encompasses the pipes and grout inside the borehole, and the ground located at radial distances to the borehole of order $r_{b}$. In the pipes, the convective transport of heat by the liquid prevails, while heat conduction takes place in the pipe walls, grout, and ground.

In the heat conduction equation to solve, the vertical heat conduction terms are of order $\Lambda^{-2}$ times smaller than the radial ones, rendering them negligible. Additionally, when the characteristic heat injection time $t_{q}$ is large compared to the characteristic transversal diffusion time $t_{b}$, the thermal inertia in the heat conduction equation is of order $t_{b} / t_{q}$ times smaller than the radial heat conduction terms, rendering it also negligible. All this allows the heat conduction problem in the inner region to be interpreted as taking place in two-dimensional planes perpendicular to the borehole, only coupled to each other through the pipes and the outer region, and in which a quasi-steady two-dimensional heat conduction problem must be solved.

Far from the borehole, at large radial distances compared to $r_{b}$, the behavior of the inner and outer regions must coincide. The boundary condition to impose there, when solving the aforementioned quasi-steady two-dimensional heat conduction problem, is given by the first two terms of the asymptotic expansion of the inner solution for $r \gg r_{b}$ (Hermanns and Pérez 2014):

$$
r \gg r_{b}: \quad T(r, z, t)=-\frac{q(z, t)}{2 \pi k} \ln \left(\frac{r}{r_{b}}\right)+T_{a}(z, t)+o(1)
$$

where $q$ is the heat injection rate per unit borehole length and $T_{a}$ is the apparent temperature at which the inner region perceives the ground. This temperature, whose value results from the asymptotic matching with the outer region, differs from the unperturbed ground temperature due to the presence of the borehole and its operation.

It was shown by Hermanns and Pérez (2014), that the heat injection rates per unit pipe length $q_{i}$, obtained as part of the solution to the quasi-steady heat conduction problem in the inner region, must obey the very specific mathematical structure 


$$
q_{1}=\frac{1+S}{2} \frac{T_{m}-T_{a}}{R_{b}}+\frac{T_{1}-T_{2}}{R_{a}}, \quad q_{2}=\frac{1-S}{2} \frac{T_{m}-T_{a}}{R_{b}}+\frac{T_{2}-T_{1}}{R_{a}}
$$

where $R_{a}$ and $R_{b}$ are respectively the thermal resistance between the pipes and the thermal resistance between the borehole and the ground. The thermal skewness parameter $S$, introduced for the first time by Ibáñez and Hermanns (2018), represents how the borehole's thermal resistance $R_{b}$ is distributed among the pipes. Its value lies in the range $[-1,1]$, corresponding the extreme values to coaxial probes.

The weighted mean fluid temperature $T_{m}$, given in terms of the temperatures $T_{i}$ of the liquid in the pipes by

$$
T_{m}=\frac{T_{1}+T_{2}}{2}-S \frac{T_{1}-T_{2}}{2}
$$

was introduced for the first time by Hermanns and Pérez (2014) and represents the temperature at which the borehole exchanges heat with the ground. This interpretation can easily be inferred from adding $q_{1}$ to $q_{2}$ :

$$
q=q_{1}+q_{2}=\frac{T_{m}-T_{a}}{R_{b}}
$$

The use of $T_{m}$ and $S$ is very convenient, because several different borehole configurations can be described using the same system of equations. For instance, single U-pipes and coaxial pipes, which in the literature are treated using different sets of equations, are treated in a unified manner here.

By substituting the expressions for $q_{i}$ into the energy conservation equations for the pipes, which are quasi-steady because of the characteristic residence time $t_{r}$ being small compared to the characteristic heat injection time $t_{q}$, a set of ordinary differential equations for $T_{1}-T_{2}$ and $T_{m}$ can be obtained, which combined with (4) constitutes the mathematical problem to solve in the inner region. Using the nondimensional variables

$$
\Delta=\frac{T_{1}-T_{2}}{Q_{c} /(\dot{m} c)}, \quad \Theta=\frac{T_{m}-T_{\text {mean }}}{Q_{c} /(k H)}, \quad \bar{q}=\frac{q}{Q_{c} / H}, \quad \Theta_{a}=\frac{T_{a}-T_{\text {mean }}}{Q_{c} /(k H)},
$$

in which $Q_{c}$ is the characteristic heat injection rate, $\dot{m}$ the mass flow rate in the pipes, $c$ the specific heat capacity of the liquid, $k$ the thermal conductivity of the ground, and $T_{\text {mean }}$ the mean annual temperature at the considered geographical location, the following system of differential and algebraic equations results (Hermanns and Ibáñez 2017; Ibáñez and Hermanns 2018):

$$
\frac{d \Delta}{d \xi}=-\bar{q}, \quad \frac{d \Theta}{d \xi}=\Omega S \bar{q}-\frac{\Omega^{2}}{A} \Delta, \quad \Theta-\Theta_{a}=\bar{q} B,
$$

where $A=k R_{a}$ and $B=k R_{b}$ are the nondimensional thermal resistances of the borehole, $\Omega=k H /(\dot{m} c)$ is a nondimensional parameter that compares the characteristic temperature difference $Q_{c} /(\dot{m} c)$ between the pipes with the characteristic temperature difference $Q_{c} /(k H)$ between the borehole and the ground located at radial distances of order $\sqrt{\alpha t_{q}}$, and $\xi=z / H$ is the nondimensional vertical coordinate.

The boundary conditions to impose at the top, $\xi=0$, and bottom, $\xi=1$, of the borehole are

$$
\xi=0: \Delta=f(\tau), \quad \xi=1: \Delta=0,
$$


being $f=Q(t) / Q_{c}$ the nondimensional heat injection rate and $\tau=t / t_{q}$ the nondimensional time.

The formulated mathematical problem requires an additional relationship between the four unknowns, $\Delta, \Theta, \bar{q}$, and $\Theta_{a}$, to be solvable. This relationship is supplied by the outer region and its asymptotic matching with the inner region.

\section{Outer region}

Far from the borehole, at radial distances of order $\sqrt{\alpha t_{q}}$, the thermal inertia of the ground becomes important, and a slender borehole is perceived in first approximation as a finite line source of heat, as inferred from the behavior of the inner region far from the borehole, given in (1) (Hermanns and Pérez 2014; Ibáñez and Hermanns 2018). Thus, the unsteady outer region is in first approximation axisymmetric, and an analytical solution for the nondimensional ground temperature, $\Theta_{g}=\left(T_{g}-T_{\text {mean }}\right) /\left(Q_{c} /(k H)\right)$, can be obtained by superposition of point sources of heat (Carslaw and Jaeger 1959):

$$
\Theta_{g}(\xi, \eta, \tau)=\Theta_{\infty}(\xi, \tau)+\frac{1}{8 \epsilon \pi^{3 / 2}} \int_{0}^{\tau} \int_{0}^{1} \frac{\bar{q}\left(\xi_{0}, \tau_{0}\right)}{\left(\tau-\tau_{0}\right)^{3 / 2}}\left[\mathrm{e}^{-\frac{\eta^{2}+\frac{\left(\xi-\xi_{0}\right)^{2}}{\epsilon^{2}}}{4\left(\tau-\tau_{0}\right)}}-\mathrm{e}^{-\frac{\eta^{2}+\frac{\left(\xi+\xi_{0}\right)^{2}}{\epsilon^{2}}}{4\left(\tau-\tau_{0}\right)}}\right] d \xi_{0} d \tau_{0}
$$

where $\eta=r / \sqrt{\alpha t_{q}}, \epsilon=\sqrt{\alpha t_{q}} / H$, and $\Theta_{\infty}$ is the nondimensional unperturbed ground temperature:

$$
\Theta_{\infty}(\xi, \tau)=G \xi+\sum_{n=-\infty}^{\infty} \widehat{T}_{s n} \mathrm{e}^{i \omega_{n} t_{q} \tau} \mathrm{e}^{-\sqrt{i \omega_{n} t} \frac{\xi}{\epsilon}}
$$

being $G=q_{g e o} H^{2} / Q_{c}$ the nondimensional geothermal heat flux at the considered geographical location and $\widehat{T}_{s n}$ the nondimensional harmonic modes, with nondimensional angular frequency $\omega_{n} t_{q}$, of the ground surface temperature oscillations with respect to the mean annual temperature $T_{\text {mean }}$.

\section{Asymptotic matching}

The asymptotic matching procedure states that both the inner and outer solutions are valid in an intermediate region defined by $r_{b} \ll r \ll \sqrt{\alpha t_{q}}$, or expressed in non-dimensional variables $(\epsilon \Lambda)^{-1} \ll \eta \ll 1$, in which their asymptotic expansions must coincide. The asymptotic behavior of the outer solution close to the borehole can be obtained by taking the limit $\eta \ll 1$ in (8). The resulting expression depends on the position along the borehole. Near the top and the bottom of the borehole, asymptotic boundary layers appear that must be analyzed separately. Also, the logarithmic singular behavior near the borehole must be extracted carefully before comparing with expression (1). Thus, the remaining non-logarithmic terms that appear in the outer solution near the borehole correspond to the nondimensional apparent temperature $\Theta_{a}$ that expressed in compact form in terms of the nondimensional heat injection rate per unit borehole length $\bar{q}$ is

$$
\Theta_{a}=F(\xi, \tau, \bar{q})
$$

All the details for the cases of steady-state excitation and time-harmonic sub-annual excitation have already been completed and submitted for publication (Hermanns and Ibáñez 2017; Ibánez and Hermanns 2018) while the case of arbitrarily time-varying heat injection rate is still under development. 


\section{Solution procedure}

The solution to the integro-differential problem formed by (6), (10), and the boundary conditions given in (7) is obtained through asymptotic expansions of the unknown variables in terms of the small parameter $\delta$ present in the problem (Hermanns and Ibáñez 2017; Ibáñez and Hermanns 2018):

$$
\Delta=\Delta_{0}+\delta \Delta_{1}+o(\delta), \quad \delta \Theta=\Theta_{0}+\delta \Theta_{1}+o(\delta), \quad \bar{q}=\bar{q}_{0}+\delta \bar{q}_{1}+o(\delta)
$$

The small parameter $\delta$ is proportional to $\log (\epsilon \Lambda)^{-1} \ll 1$ and its exact value depends on the case studied. In this expansion, the nondimensional weighted mean fluid temperature $\Theta$ is preceded by a factor $\delta$ that is foreseen by analyzing the order of magnitude of that variable in (6) (Hermanns and Ibáñez 2017; Ibáñez and Hermanns 2018). The expansions in (11) are introduced in (6) and similar order terms are grouped to obtain simpler systems of equations to be solved for each order of the expansions. The resulting analytical expressions for $\Delta, \Theta$, and $\bar{q}$ describe the thermal response of vertical geothermal boreholes and are computationally inexpensive to evaluate.

Worth to mention that the obtained apparent temperature is not uniform along the borehole, presenting deviations of around $\pm 20 \%$ with respect to a mean value. This result is important, as the mean azimuthal borehole wall temperature coincides in value with the apparent temperature (Hermanns and Pérez 2014), showing that the simplifying assumption introduced by the g-function model, that the mean azimuthal borehole wall temperature is uniform along the borehole, is not accurate.

\section{EXTENSION TO BOREHOLE FI ELDS}

Although most of the work done so far has been on single boreholes, the extension to geothermal heat exchangers comprised of tens or hundreds of boreholes has already been initiated, and preliminary results for the case of a timeharmonic sub-annual excitation will be presented for the first time in this conference.

When a time-harmonic excitation is imposed at the top of the borehole, the characteristic time of variation of the heat injection rate becomes $t_{q}=1 / \omega$, where $\omega$ is the angular frequency of the oscillation. The total heat injection rate can then be expressed as $Q(\tau)=\operatorname{Re}\left[\hat{Q} e^{i \tau}\right]$. Consequently, all variables can be expressed as harmonic functions,

$$
\Delta(\xi, \tau)=\operatorname{Re}\left[\widehat{\Delta}(\xi) e^{i \tau}\right], \quad \Theta(\xi, \tau)=\operatorname{Re}\left[\widehat{\Theta}(\xi) e^{i \tau}\right], \quad \bar{q}(\xi, \tau)=\operatorname{Re}\left[\widehat{q}(\xi) e^{i \tau}\right],
$$

and only the complex-valued harmonic modes $\widehat{\Delta}, \widehat{\Theta}$, and $\hat{q}$ need to be obtained. These complex values, that are functions of the nondimensional vertical coordinate $\xi$, include information of the amplitude of the oscillation as well as of the phase difference with respect to the total heat injection rate at any section of the borehole. A system of equations equivalent to (6) must be solved now for each borehole, and additional conditions fix the total heat injection rate $Q$ transferred to the ground by the $N_{b}$ boreholes and the common inlet temperature of the boreholes. The apparent temperature of each borehole contains now $N_{b}-1$ new terms which correspond to the temperature perturbations exerted by the remaining boreholes. These contributions are obtained by setting $\eta=B_{i j} / \sqrt{\alpha t_{q}}$ in (8), where $B_{i j}$ is the distance between the borehole and the remaining boreholes. Finally, the solution of the problem is obtained by expanding $\widehat{\Delta}$, $\widehat{\Theta}$, and $\hat{q}$ in terms of a set of $N_{b}$ small parameters as in (11). Details of the solution procedure and the resulting expansions can be found in Hermanns and Ibáñez (2018b).

A first implementation of the developed model in a computer program shows that the solution to a geothermal heat exchanger comprised of 100 boreholes can be obtained in 30 milliseconds using a single CPU core on an Intel Core i7$7700 \mathrm{~K}$ PC. Thus, the advantage of having analytical expressions for the thermal response of geothermal heat exchangers is that they can be used for the analysis of heterogeneous borehole fields with irregular placement of the boreholes without requiring the excessive computational cost of SBM or the loss in accuracy of the g-function model. 

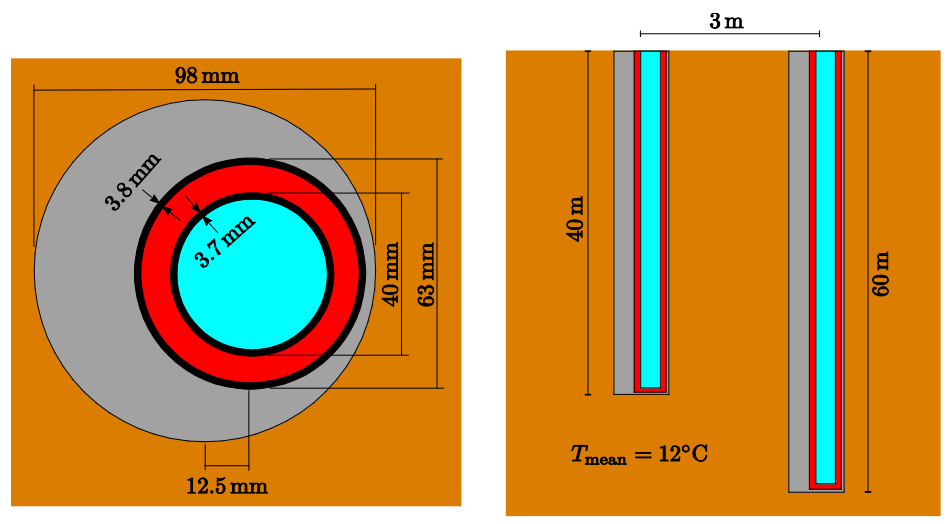

\begin{tabular}{|c|c|c|}
\hline \multirow{2}{*}{$\begin{array}{l}\text { Ground } \\
\text { (Granite) }\end{array}$} & Conductivity & $3.7 \mathrm{~W} /(\mathrm{m} \mathrm{K})$ \\
\hline & Diffusivity & $1.5 \cdot 10^{-6} \mathrm{~m}^{2} / \mathrm{s}$ \\
\hline \multirow{2}{*}{ Grout } & Conductivity & $2.2 \mathrm{~W} /(\mathrm{m} \mathrm{K})$ \\
\hline & Diffusivity & $6.79 \cdot 10^{-7} \mathrm{~m}^{2} / \mathrm{s}$ \\
\hline \multirow{5}{*}{ Liquid } & Density & $997.2 \mathrm{~kg} / \mathrm{m}^{3}$ \\
\hline & $\begin{array}{l}\text { Specific heat } \\
\text { capacity }\end{array}$ & $4180 \mathrm{~J} /(\mathrm{kg} \mathrm{K})$ \\
\hline & Conductivity & $0.608 \mathrm{~W} /(\mathrm{m} \mathrm{K})$ \\
\hline & $\begin{array}{l}\text { Dynamic } \\
\text { viscosity }\end{array}$ & $8.9 \cdot 10^{-4} \mathrm{~kg} /(\mathrm{m} \mathrm{s})$ \\
\hline & $\begin{array}{l}\text { Mass flow rate } \\
\text { (per borehole) }\end{array}$ & $0.168 \mathrm{~kg} / \mathrm{s}$ \\
\hline Pipes & Conductivity & $0.42 \mathrm{~W} /(\mathrm{m} \mathrm{K})$ \\
\hline
\end{tabular}

Figure 1 Diagram of the configuration used for the comparison of the derived asymptotic model with three existing models. (Left) Cross section of the coaxial borehole. The liquid flows downwards through the pipe shown in red and upwards through the pipe shown in cyan. The grout is shown in grey and the ground in brown. (Middle) Longitudinal section of the borehole field. Borehole 1 is at left and Borehole 2 is at right. (Right) Table of properties of the different materials used in the simulation.

\section{COMPARISON WITH EXISTING MODELS}

To assess the accuracy of the derived asymptotic expressions, comparisons with three existing models with different levels of accuracy are carried out. The first and most accurate model is a detailed numerical simulation (DNS) of the governing equations using a commercial finite-element simulation tool. The second model is the Superposition Borehole Model (SBM) developed by Eskilson and Claesson (Eskilson 1986; Eskilson and Claesson 1988). Since the original implementation is not meant for steady-state or time-harmonic excitations, a reimplementation of the method has been performed (Hermanns and Ibáñez 2017; Ibáñez and Hermanns 2018). The third model is the g-function model proposed by Eskilson (Eskilson 1987). For the steady-state case, the g-functions have been taken from the literature (Eskilson 1987; Zeng, et al. 2003; Conti 2016), while for the other regimes they have been computed using the reimplemented SBM and following the specifications given in the literature (Eskilson 1987; Eskilson and Claesson 1988; Hellström 1991; Blomberg, et al. 2017). The g-function solution has been implemented using an equally-spaced discretization with 20 segments along the boreholes, as in Cimmino and Bernier (2014).

The results from all the comparisons performed so far can be found in the work submitted for publication (Hermanns and Ibáñez 2017; Ibáñez and Hermanns 2018). Here, some preliminary results are shown for the timeharmonic sub-annual excitation of a geothermal heat exchanger comprised by two parallel coaxial boreholes of different lengths. Figure 1 contains a diagram of the borehole field studied as well as a table including the properties of all materials involved in the simulation. A total heat injection rate of modulus $|\hat{Q}|=2100 \mathrm{~W}$ and period of one year (365.25 days) is distributed among the boreholes through which a mass flow rate of $\dot{m}=0.168 \mathrm{~kg} / \mathrm{s}$ per borehole is supplied.

Figure 2 shows the modulus and argument of the complex-valued harmonic modes $\widehat{\Delta}, \widehat{\Theta}$, and $\hat{q}$ for each of the two boreholes as functions of $\xi=z / \bar{H}$, where $\bar{H}=50 \mathrm{~m}$ is the mean depth of the boreholes. The modulus of each harmonic mode is a measure of the maximum value attained as it oscillates. On the other hand, the argument of each harmonic mode is a measure of the difference in phase that appears between that variable and the total heat injection rate. 

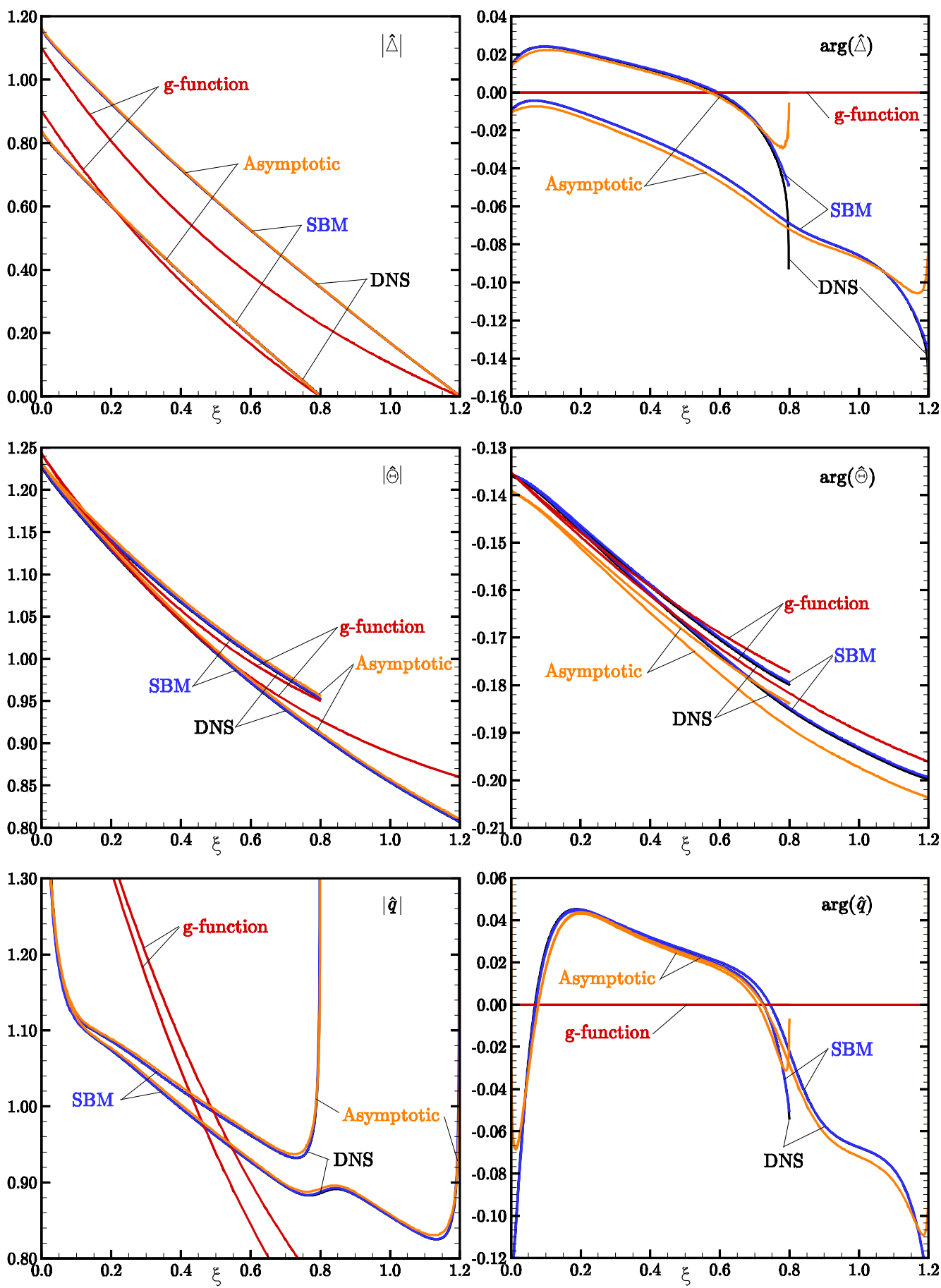

Figure 2 Time-harmonic thermal response of a geothermal heat exchanger, comprised of two heterogeneous coaxial boreholes with nondimensional depths $h$ equal to 0.8 and 1.2, to a time-harmonic heat injection rate with a oneyear period. (Left) modulus and (right) argument of the (top) nondimensional temperature difference $\widehat{\Delta}$, (middle) nondimensional weighted mean temperature $\widehat{\Theta}$, and (bottom) nondimensional heat injection rate per unit borehole length $\hat{q}$ of the two boreholes as functions of the nondimensional axial coordinate $\xi$ and for the considered models. 

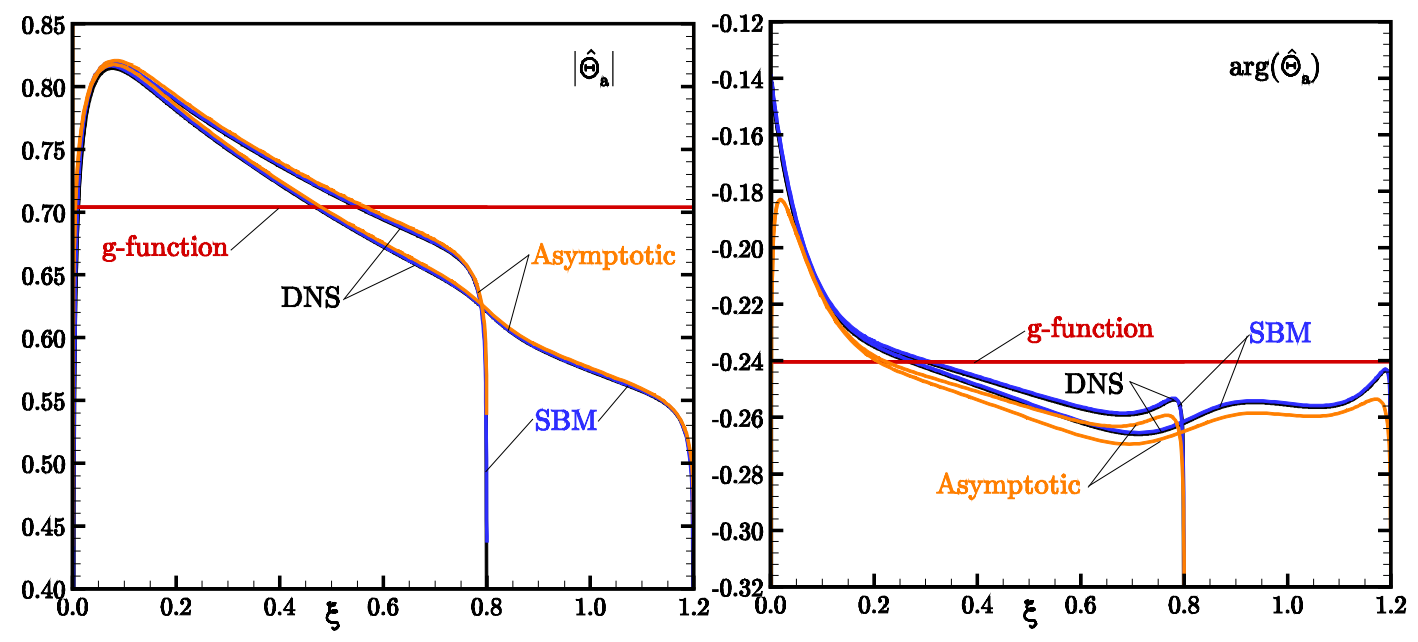

Figure 3 Time-harmonic thermal response of a geothermal heat exchanger, comprised of two heterogeneous coaxial boreholes with nondimensional depths $h$ equal to 0.8 and 1.2, to a time-harmonic heat injection rate with a oneyear period. (Left) modulus and (right) argument of the nondimensional apparent temperature $\widehat{\Theta}_{a}$ of the two boreholes as functions of the nondimensional axial coordinate $\xi$ and for the considered models.

While the developed asymptotic model, SBM, and DNS present small differences, the g-function model is unable to correctly represent the temperature and heat injection rate distributions along the boreholes. The g-function model predicts a uniform zero value of the argument of both $\widehat{\Delta}$ and $\widehat{\Theta}$ while the remaining models display notable axial variations and non-zero mean values of those variables. The most noticeable discrepancies between the g-function model and the remaining models arise in the modulus of the nondimensional heat injection rate per unit borehole $\hat{q}$. In a small region near the top and the bottom of each borehole, the nondimensional heat injection rate per unit borehole $\hat{q}$ increases abruptly (Hermanns and Ibáñez 2017). These regions, which coincide with the asymptotic boundary layers mentioned before, are correctly represented by all models except the g-function one.

Figure 3 shows the nondimensional apparent temperature $\widehat{\Theta}_{a}$ for the same configuration. The developed asymptotic model, SBM, and DNS deliver very similar results, with deviations of up to $\pm 23 \%$ with respect to a mean value. These results confirm that neither the nondimensional apparent temperature $\widehat{\Theta}_{a}$ on Figure 3 , nor the nondimensional heat injection rate per unit borehole length $\hat{q}$ on Figure 2, are uniform along the borehole. Hence, the simplifying assumptions made by Eskilson's definition of the g-function (uniform mean azimuthal borehole wall temperature) and the alternative definition found in the literature (uniform heat injection rate per unit borehole length) are not justified by the physics of the problem.

\section{CONCLUSI ONS AND FUTURE WORK}

By means of scale analysis and matched asymptotic expansion techniques, a new approach for the modeling of the thermal response of geothermal heat exchangers was initiated in 2011. A first publication, whose main focus was the asymptotic matching process and the structure of the solution to the quasi-steady heat conduction problem in the inner region, was published in 2014 (Hermanns and Pérez 2014), followed by a recent extension of that analysis to the steadystate thermal response of complete boreholes (Ibáñez and Hermanns 2018). Further work related to the time-harmonic thermal response of single boreholes (Hermanns and Ibáñez 2017) and its extension to whole borehole fields (Hermanns and Ibáñez 2018b) is in reviewing process at the moment. Also, a critical analysis of the g-function model and its different versions was recently sent for publication (Hermanns and Ibáñez 2018). In it, a mathematical explanation is given to the ill-posedness that appears in the original definition of the $g$-function model when no initial insulated length is used. 
Still in the present year, the analysis of the thermal response of single boreholes to arbitrarily time-varying heat injection rates will be finished and sent for publication. Then, in the next year, the case in which the characteristic heat injection time $t_{q}$ is comparable to the characteristic transversal diffusion time $t_{b}$ will be addressed. This regime of operation is relevant for peak loads, in which the inner region is unsteady. The same applies to the flow in the pipes in case the characteristic heat injection time $t_{q}$ is comparable to the characteristic residence time $t_{r}$. Also, the extension to borehole fields of the thermal response to arbitrarily time-varying heat injection rates will be performed.

The outcome of all this work will ultimately be a coherent theoretical framework for the thermal response of geothermal heat exchangers with irregularly placed heterogeneous boreholes, in which extensions such as stratified grounds, time-varying mass flow rates, and groundwater flow will be possible, and whose reduced computational cost will allow the implementation of automatic optimization strategies at borehole field level.

\section{ACKNOWLEDGMENTS}

Work was funded by the Spanish Ministerio de Economia y Competitividad through project ENE2015-68703-C2-2-R.

\section{REFERENCES}

Bennet, J., J. Claesson and G. Hellström. 1987. Multipole method to compute the conductive heat flows to and between pipes in a composite yylinder. Technical report of the Lund Institute of Technology. Lund, Sweden.

Blomberg, T., J. Claesson, P. Eskilson, G. Hellström and B. Sanner. 2017. EED - Earth Energy Designer 4.17. BLOCON. Lund, Sweden.

Carslaw, H. and J. Jaeger. 1959. Conduction of Heat in Solids. Oxford, UK: Oxford University Press.

Cimmino M. and Bernier M., 2014. A semi-analytical method to generate g-functions for geothermal bore fields, International Journal of Heat and Mass Transfer, 70: 641-650.

Cimmino M., Bernier M. and Adams F. 2013. A contribution towards the determination of g-functions using the finite line source. Applied Thermal Engineering, 51: 401-412.

Claesson, J. and G. Hellström. 2011. Multipole method to calculate borehole thermal resistances in a borehole beat exchanger. HVAC\&R Research 17: 895-911.

Claesson J. and Javed S. 2011. An analytical method to calculate borehole fluid temperatures for time-scales from minutes to decades. ASHRAE Transactions. 117: 279-288.

Conti P. 2016. Dimensionless maps for the validity of analytical ground heat transfer models for GSHP applications. Energies. 9: 890.

Eskilson, P. 1986. Superposition Borehole Model, Manual for Computer Code. Technical report of the University of Lund. Lund, Sweden.

Eskilson, P. 1987. Analysis of Heat Extraction Boreholes. PhD Thesis at Lund Institute of Technology. Lund, Sweden.

Eskilson, P. and J. Claesson. 1988. Simulation model for thermally interacting heat extraction boreholes. Numerical Heat Transfer 13: 149-165.

Hellström, G. 1991. Ground Heat Storage: Thermal Analyses of Duct Storage Systems, I. Theory. PhD Thesis at Lund Institute of Technology. Lund, Sweden.

Hermanns, M. and J.M. Pérez. 2014. Asymptotic analysis of vertical geothermal boreholes in the limit of slowly varying heat injection rates. SIAM Journal on Applied Mathematics 74: 60-82.

Hermanns, M. and S. Ibáñez. 2017. Thermal response of slender geothermal boreholes to sub-annual barmonic excitations. Submitted to SIAM Journal on Applied Mathematics.

Hermanns, M. and S. Ibáñez. 2018. On the ill-posedness of the g-function model for the thermal response of geothermal heat exchangers. Submitted to International Journal of Thermal Sciences.

Hermanns, M. and S. Ibáñez 2018b. Thermal response of geothermal heat exchangers to sub-annual harmonic excitations. Submitted to SIAM Journal on Applied Mathematics.

Ibáñez, S. and M. Hermanns. 2018. On the steady-state thermal response of slender geothermal boreholes, SIAM Journal on Applied Mathematics 78: 1658-1681.

Ingersoll L. R., O. J. Zobel, A. C. Ingersoll. 1954. Heat conduction with engineering, geological, and other applications. New York, USA: McGraw-Hill.

Lagerstrom, P.A. 1988. Matched asymptotic expansions: ideas and techniques. New York, USA: Springer-Verlag. 
Lamarche L. 2017. g-function generation using a piecewise-linear profile applied to ground heat exchangers. International Journal of Heat and Mass Transfer 115: 354-360.

Lamarche L. and B. Beauchamp. 2007. A new contribution to the finite line-source model for geothermal boreholes. Energy and Buildings. 39: 188-198.

Lazzarotto A. and F. Björk. 2016. A methodology for the calculation of response functions for geothermal fields with arbitrarily oriented boreholes -part 2. Renewable Energy 86: 1353-1361.

Li M. and C. K. Lai. 2015. Review of analytical models for heat transfer by vertical ground heat exchangers (GHEs): A perspective of time and space scales. Applied Energy. 151: 178-191.

Malayappan V. and J. D. Spitler. 2013. Limitations of using uniform heat flux assumptions in sizing vertical borehole heat exchanger fields. Proceedings of the Clima 2013 World Conference.

Monzó P., P. Mogensen, J. Acuña, F. Ruiz-Calvo and C. Montagud. 2016. A novel numerical approach for imposing a temperature boundary condition at the borehole wall in borehole fields. Geothermics. 56: 35-44.

Philippe M., M. Bernier and D. Marchio. 2009. Validity ranges of three analytical solutions to heat transfer in the vicinity of single boreholes. Geothermics 38: 407-413.

Zeng H., N. Diao and Z. Fang. 2002. A finite line-source model for boreholes in geothermal heat exchangers. Heat Transf Asian Res 31: 558-67.

Zeng, H., N. Diao and Z. Fang. 2003. Heat transfer analysis of boreholes in vertical ground heat exchangers. International Journal of Heat and Mass Transfer 46: 4467-4481. 